\title{
A Case of Coinfection with SARS-COV-2 and Cytomegalovirus in the Era of COVID-19
}

\author{
Damiano D'Ardes ${ }^{1}$, Andrea Boccatonda², Cosima Schiavone ${ }^{2}$, Francesca Santilli ${ }^{1}$, \\ Maria Teresa Guagnano ${ }^{1}$, Marco Bucci ${ }^{1}$, Francesco Cipollone ${ }^{1}$ \\ ${ }^{1}$ Internal Medicine, Department of Medicine and Science of Aging, 'G. D'Annunzio' University, Chieti, Italy \\ 2Ultrasound Unit, Internal Medicine, Department of Medicine and Science of Aging, 'G. D'Annunzio' University, Chieti, Italy
}

Received: 09/04/2020

Accepted: $10 / 04 / 2020$

Published: $11 / 04 / 2020$

How to cite this article: D'Ardes D, Boccatonda A, Schiavone C, Santilli F, Guagnano MT, Bucci M, Cipollone F. A case of coinfection with SARS-COV-2 and cyromegalovirus in the era of COVID-19. EJCRIM 2020;7: doi:10.12890/2020_001652.

Conflicts of Interests: The Authors declare that there are no competing interests.

Acknowledgements: The authors wish to thank the medical and paramedical staff of the 'Clinica Medica' department of Chieti Hospital who are wholeheartedly working to deal with the COVID-19 emergency with professionalism and humanity.

This article is licensed under a Commons Attribution Non-Commercial 4.0 License

\section{ABSTRACT}

The World Health Organization has declared novel coronavirus disease 2019 (COVID-19) an international public health emergency. We describe the case of a 92-year-old woman who was admitted to our unit with fever and chills with laboratory evidence of coinfection with SARS-CoV-2 and cytomegalovirus.

\section{LEARNING POINTS}

- This is the first reported case of coinfection with SARS-CoV-2 and cytomegalovirus.

\section{KEYWORDS}

COVID-19, coinfection, SARS-CoV-2, cytomegalovirus

\section{INTRODUCTION}

The World Health Organization has declared novel coronavirus disease 2019 (COVID-19) an international public health emergency and as of 5 April 2020, more than 1130000 cases have been confirmed globally ${ }^{[1]}$.

The SARS-CoV-2 outbreak in Italy began at the end of January 2020 and rapidly spread. In March we admitted approximately 200 COVID-19 patients to the University Hospital of Chieti. We have found that older people have the worst outcomes, as also shown by Chinese studies ${ }^{[2]}$.

\section{CASE DESCRIPTION}

We described the case of a 92-year-old woman who was admitted to the University Hospital of Chieti in March with a 4-day history of fever and chills. She had a history of diabetes mellitus and arterial hypertension. Her family confirmed previous contact with patients positive for SARS-CoV-2. Clinical examination in our Internal Medicine ('Clinica Medica') department revealed the patient had bilateral crackles in both lungs. She was stable haemodynamically. Arterial blood gas (ABG) analysis, blood tests and a chest x-ray were carried out. The ABG results showed mild hypoxemic hypocapnic respiratory failure, the chest x-ray showed bilateral signs of interstitial pneumonia (Fig. 1), and 
the blood tests showed lymphocytopenia, and an elevated erythrocyte sedimentation rate (42 mm), C-reactive protein (138 mg/l) and lactate dehydrogenase $(471 \mathrm{U} / \mathrm{I})$. Platelets were low $\left(54000 / \mathrm{mm}^{3}\right)$ as was procalcitonin. These findings together with the history suggested the diagnosis of SARS-CoV-2 infection.

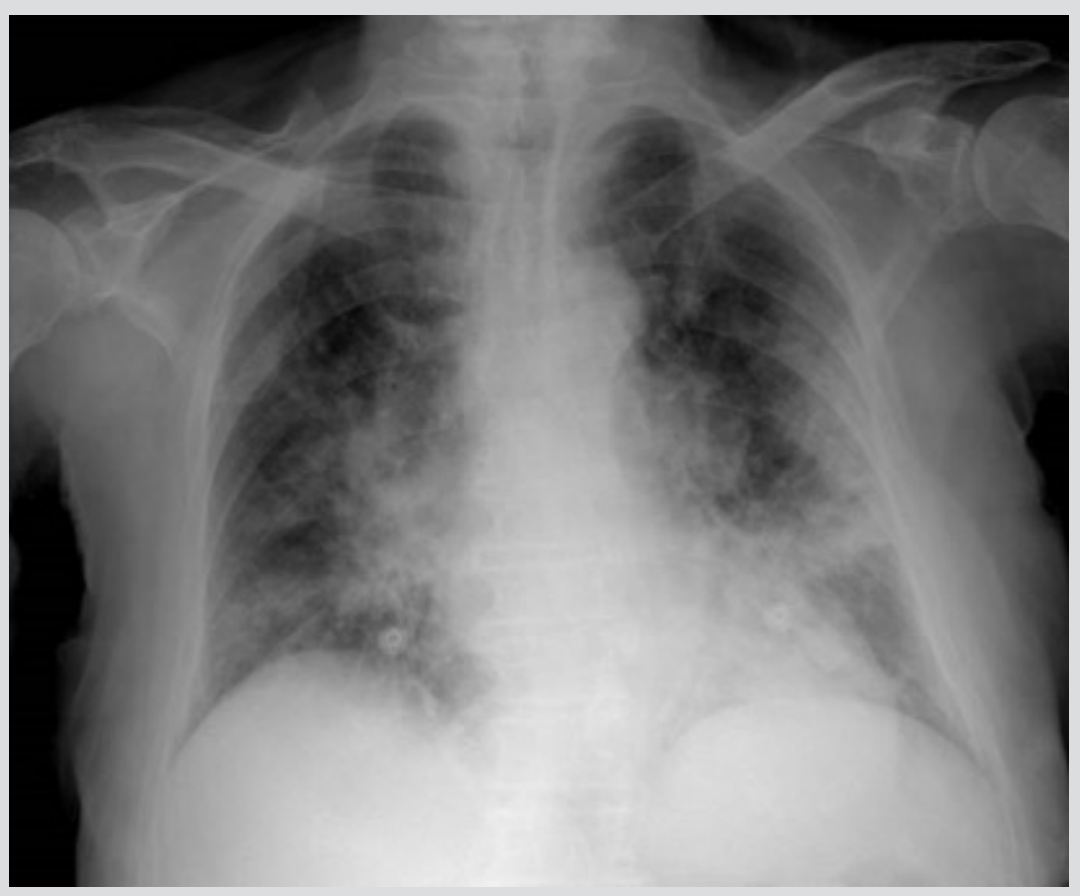

Figure 1. Chest $x$-ray showing bilateral pneumonia

The patient was tested for SARS-CoV-2 with a positive result. Tests for influenza A and B, Chlamydia pneumoniae, Mycoplasma pneumoniae, Legionella pneumophila, Herpes simplex virus and Epstein-Barr virus were all negative for recent active infection. In light of the bilateral pneumonia and lymphocytopenia, the patient was also investigated for antibodies against cytomegalovirus (CMV): results showed high IgG (>180 U/ml) and lgM (38.7 U/ml) levels, indicating recent and likely active infection.

An infectious disease specialist was consulted and prescribed empirical therapy with lopinavir/ritonavir 200 mg twice a day plus hydroxychloroquine $200 \mathrm{mg}$ twice a day.

Bronchoscopy to obtain bronchoalveolar lavage for molecular diagnosis was not possible because of the patient's unstable condition. Unfortunately, she died 6 days after admission due to severe respiratory failure in the clinical context of acute respiratory distress syndrome (ARDS).

\section{DISCUSSION}

In light of the evidence of a double viral infection (SARS-CoV-2 and CMV) in the context of bilateral pneumonia, we treated the patient with antiviral therapy. Unfortunately, she developed ARDS and died from severe respiratory failure. To our knowledge, this is first reported case of coinfection with SARS-CoV-2 and CMV. Our team have previously often found lymphocytopenia in other cases of CMV infection and reactivation, particularly in patients who had been given biological drugs. This merits investigation, as the literature ${ }^{[3]}$ suggests a link between SARS-CoV-2 and CMV infection/reactivation caused by the increasingly widespread use of anti-IL-6 and anti-IL-1 biological therapies in COVID-19.

\section{CONCLUSIONS}

COVID-19 is a global disease which is severely testing the health systems of many countries. Evidence is currently lacking for the correct management of patients. In many cases, comorbidities and coinfections coexist, complicating the clinical scenario, in particular in older people who have the worst outcomes for COVID-19. We hope that further studies will reveal new treatment opportunities to better manage these patients. 


\section{REFERENCES}

1. World Health Organization. Coronavirus disease (COVID-19) pandemic. Available from https://www.who.int (accessed 10 April 2020).

2. Zhu N, Zhang D, Wang W, Li X, Yang B, Song J, et al. A novel coronavirus from patients with pneumonia in China, 2019. N Engl J Med 2020;382(8):727-733.

3. van Duin D, Miranda C, Husni E. Cytomegalovirus viremia, pneumonitis, and tocilizumab therapy. Emerg Infect Dis 2011;17(4):754-756. 